\title{
HACIA UNA DEFINICIÓN ACTUAL DEL CONCEPTO DE «DIAGNÓSTICO SOCIAL». BREVE REVISIÓN BIBLIOGRÁFICA DE SU EVOLUCIÓN
}

\author{
Towards a current definition of the concept «social diagnosis». \\ A brief bibliographical review of its evolution
}

Silvia PATricia CURY ${ }^{1}$ y ANDrÉs Arias AstraY ${ }^{2}$

\begin{abstract}
Resumen
Presentamos en este artículo una propuesta de definición del concepto de «diagnóstico social» que consideramos contempla y sintetiza las características más destacadas señaladas por los diferentes autores que han tratado esta cuestión, desde Mary Richmond hasta la actualidad. Para ello realizamos una revisión bibliográfica de la evolución del concepto de «diagnóstico social» y el análisis de los principales elementos señalados en la literatura acerca de su correcta delimitación y formulación.
\end{abstract}

Palabras clave: «diagnóstico social», importancia metodológica, evolución histórica del concepto, delimitación, definición.

\section{Extended Abstract}

As in other care-related fields, a major professional and academic challenge for social work is appropriately defining the term "diagnosis". Its methodological importance lies in the fact that the formulation of the diagnosis must offer a full summary of the results of the study in each particular case, in order to properly ground and enable the best possible social intervention. The correct formulation of a social diagnosis in any context of social work intervention therefore represents a key challenge and an essential professional responsibility and competence.

The concept of social diagnosis has been studied, debated and argued over ever since the birth of social work. There has been considerable difficulty in properly delimiting and defining it. Moreover,

\begin{abstract}
This article presents a proposal for a definition of the concept of «social diagnosis». This definition contemplates and summarizes the salient features identified by the different authors who have dealt with this issue, from Mary Richmond to the present. A bibliographical review has been done of regarding the evolution of the concept of «social diagnosis» and there is an analysis of the main elements identified in the literature about its correct delimitation and formulation.
\end{abstract}

Key words: «social diagnosis», methodological importance, historical evolution of the concept, delimitation, definition.

formulating a social diagnosis requires the use of professional language, which has given rise to further difficulties over the course of the development of the concept. Social diagnosis has been considered, on one hand, "the hallmark of social work", and on the other, a professional "weakness" to be overcome and which may be understood as comprising the most complex methodological phase in social work.

We may also recall that, as stated by various authors, the concept of social diagnosis has been given many other names: "assessment", "evaluation", "technical opinion", "diagnostic judgment", "professional judgment", "diagnostic interpretation", "technical-scientific assessment" and "professional opinion", among others. Nevertheless, the need to have as much accurate knowledge as possible regarding the nature of social situations

Recibido: 05/10/2015 Aceptado: 14/03/2016

Publicado: 20/12/2016

1. Facultad de Trabajo Social, Universidad Complutense de Madrid. C/ Palafox 23. Piso 2. Puerta 4. 28010. Madrid.spcury@ucm.es

2. Facultad de Trabajo Social, Universidad Complutense de Madrid. 
requiring intervention has always been present in the discipline.

A concept as important as social diagnosis will remain the object of analysis and debate in social work, as in other care-related disciplines. All of these disciplines will also continue to need to act on the basis of existing knowledge at any given time and, hence, to increase such knowledge by formalizing and disseminating it. In this regard, we provide a review of the literature regarding the evolution of the concept of "social diagnosis" and an analysis of the main elements identified in the literature with regard to its delimitation and formulation. On the basis of this analysis, we offer a proposed definition of the concept of "social diagnosis" that, in our view, contemplates and summarizes the key features identified by the various authors who have addressed this issue, from Mary Richmond to the present day.

The aim of producing a proposed definition of the concept of "social diagnosis" results from its methodological importance, as well as the possibilities offered by its current development and the need to summarize the main elements identified in the literature as fundamental in properly formulating a social diagnosis. It is worth noting that considerable efforts have been made in the discipline to overcome the difficulties in defining and delimiting social diagnosis since the 1980s, resulting in the design of specific instruments to formulate social work diagnoses.

It is also striking that in Spain, a considerable level of knowledge and professional consensus has been achieved in professional and legal terms with regard to social diagnosis. In this respect, 15 of the 17 social services laws established by the Spanish autonomous communities state that social diagnosis is a citizen's right and one of the basic provisions of the public social services system. These laws also establish that social diagnosis will be the result of a comprehensive evaluation of the user's needs, enabling the production of an individualized social care plan and selection of suitable resources and provisions, in terms of both general and specialist social services, in each case.

In turn, the Spanish Social Work Code of Conduct (Código Deontológico del Trabajo Social, Consejo General del Trabajo Social, 2012) identifies social diagnosis as the phase carried out by social workers prior to intervention, establishing that professionals will record the diagnosis for each case in the social history report and that the design of any social intervention will include an evaluation and diagnosis of the situations and individuals involved in that intervention.

In recent decades, the conceptual and methodological development of social diagnosis in Spain has represented an important advance in the discipline, more so as it has been backed by the legal implementation of the concept. However, it remains an initial advance in need of subsequent development. On occasion, there is even a certain degree of conceptual confusion between the process of investigating or assessing a social situation and that of social diagnosis, even if one may consider that the term "social diagnosis" is increasingly widely accepted and enjoys an ever-higher degree of professional and legal consensus. For the above reasons, we consider it to be worthwhile and important to propose a current definition of the concept of "social diagnosis", the objective of which is to summarize and delimit its main characteristics.

The definition covers the main conceptual elements identified in the literature analysed with relation to the concept of "social diagnosis". In this respect, social diagnosis has been defined as a professional judgment which the social worker bears responsibility for formulating. A distinction has been drawn between social diagnosis in the study phase and social diagnosis in the social intervention phase. As such, social diagnosis represents the fundamental methodological nexus between the two phases, and should be formulated with the highest degree of precision and correctness permitted by the knowledge of the discipline at any given time. Moreover, the definition that we propose responds to the importance of social diagnosis contemplating both difficulties and strengths in a particular situation, for purposes of achieving the best social intervention for that situation. The definition also establishes that the social diagnosis will take into account both the individual situation and the family, social and institutional environment of the individual, since the latter constitutes a fundamental element in the diagnosis, social intervention and prognosis of the situation.

Lastly, it is worth noting that the definition we propose should be considered a further step in the process of delimiting the concept of "social diagnosis". The aim of our contribution is to add our efforts to the interest and investment already made in social work to achieve the greatest possible level of specificity with respect to the concept and application of social diagnosis within the intervention process. 


\section{Introducción}

En trabajo social, la definición adecuada del término «diagnóstico» constituye un desafío profesional y académico de primer orden, al igual que ocurre en las demás disciplinas asistenciales. Su relevancia metodológica radica en que la formulación del diagnóstico ha de ofrecer una síntesis completa de los resultados del estudio de cada caso particular, que permita y fundamente de manera adecuada la mejor intervención social posible. Por lo tanto, la correcta formulación del diagnóstico social en cualquier ámbito de intervención en trabajo social supone un reto destacado, y constituye una responsabilidad y una competencia profesional propia.

El concepto de diagnóstico social ha sido objeto de estudio, debate y controversia permanentes desde el nacimiento del trabajo social. Su correcta delimitación y definición han presentado una considerable dificultad. Su formulación requiere, además, la utilización de un lenguaje profesional propio, y ésta ha sido otra de las dificultades halladas en el desarrollo histórico del concepto. El diagnóstico social se ha considerado, por una parte, «seña de identidad del trabajo social» (Colom, 2009a, p. 139); por otra, una «debilidad» profesional que ha de ser superada y que puede ser comprendida, precisamente, por constituir la fase de mayor complejidad del método en trabajo social (Gobierno Vasco, 2006, p. 77).

Recordemos además que, tal como señalaron varios autores (Colom, 2009a; Gobierno Vasco, 2006; Hamilton, 1946; Perlman, 1968; Woods y Hollis, 1964), el concepto de diagnóstico social ha recibido múltiples denominaciones alternativas: «valoración», «evaluación», «dictamen técnico», «juicio diagnóstico», «juicio profesional», «interpretación diagnóstica», «valoración técnico-científica», «opinión profesional», etc. No obstante, la necesidad de contar con el conocimiento más amplio y preciso posible acerca de la naturaleza de las situaciones sociales objeto de intervención ha estado siempre presente en la disciplina (Ander Egg y Aguilar, 2001; Colom, 2009a, 2009b, 2012; Escalada, Fernández y Fuentes, 2001; Escartín, Palomar y Suárez, 1997; García, 2008; Greenwood, 1955; Hamilton, 1923, 1946; Martín, Miranda y Suárez, 1996; Pelton, 1910; Richmond, 1917/2007, 1917, 1930; Perlman, 1957; Scarón de Quintero, 1974; Selby, 1958; Turner, 1968, 1974, 2002; Woods y Hollis, 1964).

Un concepto tan importante como el del diagnóstico social continuará siendo objeto de análisis y debate en trabajo social, como ocurre en las demás disciplinas asistenciales. No en vano, en todas ellas también permanecerá inalterada la necesidad de actuar sobre la base del conocimiento existente en un momento dado, y la de ampliar dicho conocimiento a través de su 
formalización y publicidad. Por ello, el objeto de este artículo es presentar una definición del concepto de diagnóstico social que reúna y sintetice los principales elementos señalados en la literatura acerca de su naturaleza y correcta delimitación. Mostramos para ello, en primer lugar, el análisis de la evolución histórica del concepto de «diagnóstico social»y, a continuación, la definición que proponemos y su justificación.

\section{El concepto de «diagnóstico social». Revisión bibliográfica}

La célebre obra de Richmond (1917a/2007), «Diagnóstico social», hizo famosa la denominación de este concepto hace casi un siglo, y marcó el inicio de un largo recorrido histórico en el que el concepto de diagnóstico social ha sido debatido, discutido, analizado y desarrollado. Richmond (1917b) afirmó que la palabra «diagnóstico» era el término adecuado para denominar la fase final del proceso de estudio y de la investigación de las circunstancias sociales de un caso. Al témino «diagnóstico» Richmond añadió el adjetivo «social»: «el criterio de lo social, su elemento siempre indispensable, es la influencia de unas mentes sobre otras» (Richmond, 1917b, p. 112). Pelton (1910, p. 332) había señalado la importancia del diagnóstico social como fase metodológica previa al "pronóstico y el tratamiento» de las necesidades de los enfermos sin recursos en el ámbito de la atención hospitalaria. Pero fue Richmond quien propuso la primera definición explícita del concepto:

«Mediante un diagnóstico social, se trata de llegar a una definición lo más exacta posible de la situación social y la personalidad de un cliente dado. El proceso comienza con la investigación o la recopilación de la evidencia, prosigue con su análisis crítico y finaliza con su interpretación y la definición de la dificultad social. En general, los trabajadores denominan investigación a todo el proceso, pero como su principal defecto es no prestar la debida atención a los procesos de comparación e interpretación y darle una excesiva importancia al etapa de recolección de la evidencia, resulta más instructivo para definir todo el proceso usar la palabra que describe, en concreto, el final del proceso» (Richmond, 1917a/2007, pp. 34-35).

Más adelante en esta misma obra, Richmond describió con mayor detalle el sentido y el contenido del diagnóstico social:

«El diagnóstico social puede describirse como la tentativa de realización de una definición lo más exacta posible de la situación y personalidad de un ser humano que presenta una dificultad social, de su situación y personalidad en relación con otros seres humanos de los que, de algún modo depende o que dependen de él, y en relación también con las instituciones sociales de su comunidad» (Richmond, 1917a/2007, p.420). 
El trabajo pionero de Richmond ha marcado en buena medida la orientación del trabajo social con casos. Su interés por defender la especificidad y la profesionalización de la disciplina (1917b, p. 113) ha influido en el desarrollo del trabajo social y de algunos de los principales modelos de intervención a lo largo de un siglo. Así, Hamilton (1923) describió la importancia decisiva de la realización de un diagnóstico social útil, que permitiera una intervención eficaz en cada caso, y expresó la necesidad de desarrollar métodos de investigación y de diagnóstico más elaborados, así como mejores sistemas de registro de la información. En 1946, Hamilton, en su obra «Principles of social work recording», continuó desarrollando el concepto de diagnóstico social, y señaló que este término no abarcaba únicamente la comprensión de un problema, sino también la comprensión de la persona que tiene el problema. Esta autora propuso que el diagnóstico social es «una opinión profesional sobre la naturaleza real del problema que el cliente presenta» (Hamilton, 1946, p. 82.) y señaló la existencia de algunas de las principales dificultades que la formulación del diagnóstico social había encontrado para su plena implantación y desarrollo en trabajo social. Entre ellos, destacó la resistencia de los profesionales para formular por escrito el diagnóstico social, la confusión existente entre la fase de investigación y la fase de diagnóstico, así como la profusión de diferentes términos para designar dicho diagnóstico. Hamilton señaló la importancia de la correcta formulación del diagnóstico social como método para superar formulaciones precipitadas, generalizaciones e imprecisión. Por otra parte, hizo especial hincapié en la trascendencia de distinguir entre la fase de investigación y la de la formulación del diagnóstico social. La destacada obra de esta autora culminó con el desarrollo de la «primera forma del casework social» (Du Ranquet, 1996) que se constituyó como «El Modelo diagnóstico o psicosocial» (Hamilton, 1951). Este modelo se fundamentó tanto en el concepto de «persona en situación» como en el de «diagnóstico social», del que tomó su denominación. Según Hamilton (1951) el diagnóstico social consiste en realizar un examen crítico tanto de la situación de la persona como del problema que presenta, así como de su entorno físico, económico, social y cultural.

En la década de los 30, bajo la influencia de la teoría de la voluntad de Otto Rank (1936), el desarrollo del «Modelo funcional» (Robinson, 1930) puso el acento en los aspectos psíquicos de la persona y en su voluntad para afrontar las dificultades, cuestionando la importancia del diagnóstico dentro del proceso de ayuda en trabajo social. No obstante, el concepto de diagnóstico social recibió una gran atención, especialmente en el contexto del trabajo social norteamericano durante las siguientes décadas. En este sentido, 
Greenwood (1955) y Selby (1958) señalaron la necesidad de que el trabajo social desarrollara clasificaciones de diagnóstico social, destacando su utilidad para sistematizar el conocimiento de la disciplina. Hollis (1954) defendió una concepción psicosocial del diagnóstico, que contemplaba tanto la situación social como la personalidad individual. Para esta autora, un diagnóstico social completo debía tener en cuenta tanto la etiología del problema como la información detallada acerca de la situación vital de la persona, de su actitud frente al problema en cuestión y de las diferentes fortalezas y oportunidades implicadas en la situación. Woods y Hollis (1964) fueron las principales impulsoras del desarrollo del «Modelo psicosocial» que constituyó, en cierto sentido, una evolución del «Modelo diagnóstico o psicosocial» de Hamilton (1951). Woods y Hollis (1964) identificaron en la obra de esta última las principales dificultades existentes en torno al concepto y a la utilización del diagnóstico social. Así, destacaron la frecuente confusión entre los términos «evaluación» y «diagnóstico», que en ocasiones eran utilizados por los profesionales de forma indistinta. Por otra parte, señalaron la resistencia de algunos trabajadores sociales frente al término "diagnóstico», que asociaban con una cierta adscripción del trabajo social a la medicina. La preocupación de los profesionales hacía referencia al riesgo de que, bajo la influencia de esta última, el diagnóstico social hiciera mayor hincapié en las debilidades que en las fortalezas y capacidades de la persona para hacer frente a sus dificultades. Woods y Hollis (1964) describieron también la preocupación profesional acerca de la utilización de «etiquetas» en la formulación del diagnóstico social, cuyo efecto indeseado pudiera ser tanto la estigmatización como el uso de estereotipos en dicha formulación. Estas autoras señalaron la importancia de que el diagnóstico social contemplara la situación de la persona en el entorno social en el que vivían y, por lo tanto, de las potenciales oportunidades que dicho entorno implicaba, así como la trascendencia de la participación activa de la persona en la elaboración de su propio diagnóstico social. Woods y Hollis señalaron que, efectivamente, el diagnóstico social siempre debía contemplar tanto las fortalezas como las debilidades de la persona, de su familia y de la situación social, así como la evaluación de las interacciones habituales entre la persona y todos los demás elementos del sistema y del medio ambiente del que la persona forma parte. Al mismo tiempo, señalaron la necesidad y la urgencia de desarrollar una tipología sistemática que clasificara los diferentes problemas objeto de diagnóstico y de intervención en trabajo social. En este sentido, en nuestro entorno, Ituarte (1992) ha realizado una importante aportación respecto de la complejidad, los límites y la importancia de las clasificaciones en el ámbito del trabajo social sanitario. En su obra «Procedimiento y proceso en 
Trabajo Social Clínico» (Ituarte, 1992, p.32) describe la dificultad de establecer categorías exactas de diagnóstico social, dada la multiplicidad de factores en interacción en una situación social dada, y el diferente valor e importancia que cada uno de estos factores tienen en dicha situación. Pero más allá del carácter único de cada caso individual, Ituarte señala la importancia de la tarea de clasificación en trabajo social que permita un análisis riguroso de las diferentes situaciones y variables objeto de diagnóstico y de intervención en el ámbito socio-sanitario.

Turner $(1968,1974)$ defendió, en este sentido, la utilización del término y del concepto de «diagnóstico» y destacó que el diagnóstico social, y las categorías empleadas para su formulación, no implicaban exclusivamente la identificación con el modelo médico asistencial, ni excluían la participación de la persona en el proceso de la intervención social. Para este autor, el término diagnóstico implicaba para el trabajador social el acto conceptual de formular un juicio del que se hacía responsable profesionalmente, y sobre cuya base se llevaba a cabo la intervención social. En esta misma línea, Perlman (1968, p. 18) consideró que el trabajo social no contaba todavía con un verdadero desarrollo del diagnóstico social puesto que, en sus propias palabras, «el diagnóstico implica la existencia de un sistema de clasificación». Esta autora describió la situación del diagnóstico social, destacando los cambios que el concepto había ido experimentando desde la célebre definición de Mary Richomnd (1917a/2007). Perlman (1968) subrayó la confusión de los trabajadores sociales, y de las principales escuelas de pensamiento en trabajo social, acerca de cómo debía formularse un diagnóstico social y de cuál era la verdadera naturaleza del concepto, así como el desconocimiento de en qué medida el diagnóstico social realmente contribuía de una manera eficaz a fundamentar la intervención. Perlman (1957) fue la gran impulsora del «Modelo de resolución de problemas» y propuso el concepto de «rol social» para delimitar el campo específico del conocimiento y de la práctica en trabajo social. El concepto de rol social se adecúa, en opinión de Perlman, a la naturaleza del trabajo social puesto que, de este modo, la atención del profesional se centra en la persona que se encuentra en algún tipo de situación que le supone una interacción problemática respecto de las demandas y las expectativas de las personas y de las condiciones de su entorno social (Perlman, 1968). Los elementos fundamentales para la delimitación del concepto de diagnóstico social son, por una parte, el concepto de «rol social»y, por otra, la motivación, la capacidad y las oportunidades del ser humano. El concepto de «rol social» aporta al diagnóstico el necesario enfoque social y relaciona al ser humano con todas las interacciones sociales en las que se encuentra implicado y en las 
que el hombre se expresa y se realiza a sí mismo. Por ello, Perlman propone que la resolución de un determinado problema social sea alcanzada a través del diagnóstico de las dificultades presentes en el desempeño, satisfactorio y eficaz, de uno o varios roles sociales, y tomando en consideración las fortalezas, la motivación y la capacidad personal.

Sainsbury (1970) definió el diagnóstico social como un proceso cuyo objetivo es el descubrimiento de patrones de significado a través del análisis y la interpretación de la información obtenida de la persona o inferida por el profesional. Este autor señaló que el principal valor del diagnóstico social estriba, fundamentalmente, en la relevancia que éste posee para los siguientes elementos de la intervención social: «a) la solución del problema social, b) las necesidades materiales y emocionales del cliente, c) la capacidad de la entidad y del trabajador social para ayudar a la persona» (Sainsbury, 1970, p. 17). En este sentido, Sainsbury analizó el concepto de diagnóstico sobre la base fundamental de su utilidad y de su relevancia dentro del proceso de ayuda. Este autor coincidió con Timms (1964) en la importancia concedida a la participación de la persona en dicho proceso, y destacó que el diagnóstico social no debe ser nunca considerado como un proceso terminado, puesto que se modificará en función de las cambiantes circunstancias a lo largo de la intervención social.

A partir de los años 70, varios autores hispanoamericanos expresaron su acuerdo con la consideración del diagnóstico social como la síntesis final del proceso de investigación, que debe ser formulado explícitamente como fundamento de la intervención social (Ander Egg y Aguilar, 2001; Colom, 2009a, 2009b, 2012; Escalada, Fernández y Sotos, 2001; García, 2008, Martín, Miranda y Suárez, 1996; Scarón de Quintero, 1974). Estos autores expresaron un elevado grado de preocupación por el insuficiente desarrollo teórico y metodológico que había tenido el diagnóstico social, y destacaron la gravedad de esta situación, en función de la importancia y la influencia de la correcta formulación de dicho diagnóstico como fundamento de la intervención social.

Consideramos, como conclusión del análisis arriba expuesto, que el diagnóstico social es la fase final del estudio de una determinada situación, que debe ser formulada explícitamente por el trabajador social, a fin de fundamentar adecuadamente la intervención. Por todo ello, la correcta formulación del diagnóstico social en cualquier ámbito de intervención en trabajo social constituye un reto profesional destacado y la propuesta de definición del concepto que realizamos en este artículo pretende dar continuación al esfuerzo disciplinar realizado en trabajo social desde sus inicios, y constituir un 
eslabón más del debate acerca de la naturaleza y las principales características del diagnóstico social.

\section{Propuesta de definición del concepto de «diagnóstico social»}

El objetivo de formular una propuesta de definición del concepto de «diagnóstico social» responde a la importancia metodológica de este concepto, así como a las posibilidades que ofrece el desarrollo actual del mismo, y a la necesidad de sintetizar los principales elementos señalados en la literatura como fundamentales para la correcta formulación del diagnóstico social. Consideramos que muchos de los obstáculos que la definición del diagnóstico social ha encontrado a lo largo de la historia han sido superados o se encuentran en proceso de superación. Cabe destacar que, a partir de los años 80, se ha realizado un considerable esfuerzo disciplinar para superar las dificultades descritas acerca de la definición y la delimitación del diagnóstico social, que ha permitido el diseño de instrumentos específicos para la formulación del diagnóstico en trabajo social (Colom, 2009a; Cury, 2009; Cury, Arias y Palacios, 2015; Gobierno Vasco, 2006, 2011, 2012a, 2012b, 2012c, 2012 d, 2012e, 2012f, 2012g; Karls y O'Keffe, 2008; Karls y Wandrei, 2008; Martín, Miranda y Suárez, 1996).

En la actualidad, la práctica del Trabajo Social se fundamenta en un conjunto de teorías tanto propias y específicas de la disciplina, como aquellas que comparte con otras Ciencias Sociales. Este amplio conjunto de teorías orientan la elección de diferentes perspectivas paradigmáticas y modelos de intervención en trabajo social, empleándose en la práctica una gran variedad de modelos, que abarcan desde aquéllos que ponen el acento en los aspectos más individuales de la situación social hasta aquéllos que se fundamentan en la importancia de la interrelación de la persona, los grupos y las comunidades. Estos modelos se enmarcan en diferentes perspectivas paradigmáticas, clasificadas y denominadas de diferente modo por distintos autores: psicosocial, conductual y crítica (Barranco, 2007), psicodinámicas; de intervención en crisis y centrados en la tarea; conductuales; de sistemas y ecológicos; sociopsicológicos y de comunicación; humanistas y existenciales; cognitivos; radicales y marxistas; promotores de la potenciación y la defensa, y anti-opresivos (Adams, Dominneli y Payne, 2002; Brown, 1992; Campanini y Luppi, 1991; Du Ranquet, 1996; Payne, 1995 Rubin y Babbie, 1997).

La definición del concepto de diagnóstico social que proponemos hace hincapié en la necesidad de que el diagnóstico contemple tanto las dificultades como las fortalezas de la persona y de su situación individual, familiar, social e institucional. Por ello consideramos que nuestra definición se 
compadece con los planteamientos teóricos del Paradigma Piscosocial, que se fundamenta en la importancia de los diferentes aspectos de la situación de una persona y en la interacción entre ésta y su contexto. Desde este enfoque, que hemos escogido como marco conceptual de la definición propuesta, la práctica profesional se orienta a potenciar las capacidades y fortalezas de la persona, pero también hacia el medio social para facilitar los medios y realizar las modificaciones necesarias de las políticas de bienestar social que permitan atender las posibles dificultades y favorecer el desarrollo humano y sus capacidades (Barranco, 2007).

Cabe señalar, además, que en el ámbito profesional y jurídico español se ha alcanzado un considerable grado de conocimiento y de consenso profesional acerca del diagnóstico social. En este sentido, 15 de las 17 comunidades autónomas españolas establecen en sus leyes de servicios sociales que el diagnóstico social constituye uno de los derechos específicos del ciudadano y una de las prestaciones básicas del sistema público de servicios sociales. Estas leyes establecen, además, que el diagnóstico social será el resultado la evaluación integral de las necesidades del usuario, que permitirá orientar el plan individualizado de atención social y la elección de los recursos y las prestaciones idóneos en cada caso, tanto a nivel de los servicios sociales generales como especializados.

En este sentido, el diseño del «Modelo global de diagnóstico» (Gobierno Vasco, 2012b) constituye, en nuestra opinión, el ejemplo más actual y destacado de la evolución profesional y jurídica del diagnóstico social como elemento metodológico fundamental dentro del proceso de intervención en trabajo en social en España. Este ambicioso diseño responde al cumplimiento de la «Ley 12/2008, de 5 de diciembre, de Servicios Sociales del Gobierno Vasco». A fin de garantizar el cumplimiento y la homogeneidad del procedimiento básico de atención social, la citada Ley establece que todas las personas que se encuentren en la Comunidad Autónoma tendrán derecho a los servicios de información, valoración, diagnóstico y orientación, y a la gestión pública de estos servicios, que serán prestados en un plazo de tiempo razonable (Ley 12/2008 de Servicios sociales, arts. 4; 60 y 9). Para ello, la Ley regula la implementación de instrumentos técnicos comunes que garanticen la homogeneidad en los criterios de intervención en los servicios sociales. En cumplimiento de la Ley, el «Decreto 353/2013, de 28 de mayo» aprobó el diseño de la «Ficha social» y del «Instrumento de diagnóstico social del Sistema Vasco de Servicios Sociales» y establece el carácter obligatorio de estos instrumentos. No se incluyen instrumentos o modelos que regulen el plan de atención personalizado, que se diseñará en función del resultado del diagnóstico 
social. Por ello, se ha utilizado la denominación «más precisa de diagnóstico social» (Decreto 353/2013, de 28 de mayo, de Ficha Social del Sistema Vasco de Servicios Sociales y del instrumento de diagnóstico social del Sistema Vasco de Servicios Sociales, p. 3), que permitirá valorar con criterios comunes la situación de la persona usuaria, y determinar la forma de diseñar el plan de intervención. Por lo tanto, el Decreto regula la utilización del «Modelo global de diagnóstico social» (Gobierno Vasco, 2012b) como un conjunto integrado de instrumentos que cuenta con los «Instrumentos comunes de diagnóstico social» (Gobierno Vasco, 2012a), cinco manuales de terminología y criterios y 171 indicadores específicos (Gobierno Vasco, 2012c, 2012d, 2012e, 2012f, 2012g). Consideramos que el ejemplo que hemos descrito ilustra de manera clara el grado de desarrollo e implantación del diagnóstico social dentro del proceso de intervención en trabajo social, su importancia, y la reglamentación jurídica de la que es objeto en la actualidad en España.

A su vez, el Código Deontológico del Trabajo Social (2012) identifica el diagnóstico social como la fase previa a la intervención que realizan los trabajadores sociales, establece que los profesiomales consignarán el diagnóstico de cada caso en la historia social y que el diseño de toda intervención social constará de una evaluación-diagnóstico de las situaciones y de las personas implicadas en dicha intervención.

En las últimas décadas, el proceso de desarrollo conceptual y metodológico del diagnóstico social en España ha consituido un importante avance disciplinar; avalado además por la implantación jurídica del concepto. No obstante, se trata de un avance inicial que requiere un desarrollo posterior. Existe aún, en ocasiones, cierto grado de confusión conceptual entre el proceso de investigación o de valoración de una situación social y el de diagnóstico social, si bien puede considerarse que la implantación del término «diagnóstico social» es cada vez más amplia y goza de mayor grado de consenso profesional y jurídico. Por todo ello consideramos relevante y justificada la propuesta de una definición actual del concepto de «diagnóstico social», cuyo objetivo es resumir y delimitar sus principales características, tal como mostramos y analizamos a continuación.

El diagnóstico social es el juicio profesional que formula el trabajador social como resultado del estudio y de la interpretación de una situación social dada y que constituye el fundamento de la intervención social en dicha situación. La correcta formulación del diagnóstico social es responsabilidad y competencia del trabajador social, y deberá contemplar las dificultades y las fortalezas de la persona y de su situación individual, familiar, social e institucional. 
Esta definición responde a los principales elementos conceptuales identificados en la literatura analizada en relación con el concepto de «diagnóstico social». De este modo, se ha definido el diagnóstico social como un juicio profesional cuya formulación compete y es responsabilidad del trabajador social, y se ha diferenciado el diagnóstico social de la fase de estudio y de la fase de intervención social. Así, el diagnóstico social constituye el nexo metodológico fundamental entre ambas fases, y deberá ser formulado con la mayor precisión y corrección que el conocimiento de la disciplina permita en un momento dado (Ander Egg y Aguilar, 2001; Colom, 2009a, 2009b, 2012; Escalada, Fernández y Soto, 2001; García, 2008; Hamilton, 1923, 1946; Martín, Miranda y Suárez 1996; Pelton, 1910; Richmond, 1917/2007; Scarón de Quintero, 1974; Turner, 1968, 1974, 2002). Por otra parte, la definición propuesta responde a la importancia de que el diagnóstico social contemple las dificultades pero también las fortalezas presentes en una determinada situación a efectos de alcanzar la mejor intervención social en dicha situación (De la Paz, 2011; Early y Glenmayer, 2000; Hamilton, 1951; Khistardt, 1994; Martín, Miranda y Suárez, 1996; Perlman, 1957; Rapp, 1998; Richmond, 1930; Salebeey, 1992, 1996; Weick, Rapp, Sullivan y Kisthardt, 1989; Woods y Hollis, 1964) Asismismo, la definición establece que el diagnóstico social considerará tanto la situación individual de la persona como la de su entorno familiar, social e institucional puesto que éste constituye un elemento fundamental para el diagnóstico, la intervención social y el pronóstico de la situación (Hamilton, 1951; Hollis, 1954; Richmond, 1917/2007; Sainsbury, 1970; Woods y Hollis, 1964).

Por último, cabe señalar que la definción que proponemos ha de ser considerada un paso más en el proceso de delimitación del concepto de «diagnóstico social». El objetivo de nuestra aportación es sumarnos al interés y al esfuerzo disciplinar realizado históricamente en trabajo social por alcanzar la mayor concreción posible respecto del concepto y la aplicación del diagnóstico social dentro del proceso de intervención.

\section{Referencias bibliográficas}

Adams, R. Dominelli, L. y Payne, M. (2002) (Eds.) Social Work. Themes, issues and critical debates. New York: Plagrave and Open University.

Ander EgG, E. y Aguilar, M. J. (2001). Diagnóstico Social. Conceptos y Metodología. Buenos Aires: Lumen.

BARRANCO, C. «La construcción del conocimiento y visión de las perspectivas paradigmáticas y teorías aplicadas en los modelos de Trabajo Social.» Servicios Sociales y Política Social 80 (2007): 65-79. 
BROWN, J. (1992). Handbook of Social Work Practice. Illinois: Charles C. Thomas Publisher.

Campanini, A., \& LupPI, F. (1991). Servicio Social y Modelo Sistémico. Una nueva perspectiva para la práctica cotidiana. Barcelona: Paidós.

Colom, D. (2009a). La investigación sobre el diagnóstico social sanitario: trabajo de campo, resultados y conclusiones. Revista de Trabajo Social y Salud., 62, 129-389.

Colom, D. (2009b). Procedimientos sociales sanitarios y diagnóstico social sanitario: Caminos de innovación en el Trabajo Social sanitario. Conferencia $X$ Congreso Nacional de la Asociación de Trabajo Social y Salud. Oviedo.

Colom, D. (2012). El diagnóstico social sanitario. Barcelona: Universidad Oberta de Catalunya.

Colomer, M. (1974). Método de Trabajo Social. Revista de Trabajo Social, 31-35.

Consejo General del Trabajo Social (2012). Código Deontológico del Trabajo Social. Aprobado en Asamblea General de Colegios Oficiales de Diplomados en Trabajo Social y Asistentes Sociales el 9 de junio de 2012. Madrid: Consejo General del Trabajo Social.

CURY, S. (2009). Estudio del diagnóstico social en residencias para personas mayores asistidas de la Comunidad de Madrid: diseño de un instrumento de valoración y diagnóstico social. Cuadernos de Trabajo Social, 22, 201-227.

CURY, S., ARIAS, A. y PALACiOS, J.L. (2015). Diseño y validación de un instrumento para el diagnóstico social en residencias para personas mayores del servicio regional bienestar social de la Comunidad de Madrid. Obtenido de Universidad Complutense de Madrid. Catálogo Cisne: http://goo.gl/iKunre

Decreto 353/2013, de 28 de mayo, de Ficha Social del Sistema Vasco de Servicios Sociales y del instrumento de diagnóstico social del Sistema Vasco de Servicios Sociales, Boletín Oficial del País Vasco, 121, de 26 de junio de 2013.

DE LA PAZ, P. (2011). La intervención en Trabajo Social desde la perspetiva de las fortalezas. Cuadernos de Trabajo Social, 24, 155-163.

DU RANQUET, M. (1996). Los modelos en trabajo social. Intervención con personas y familias (1. ${ }^{\text {a }} 1981$ ed.). Madrid: Siglo XXI.

EARLY, T. y GLENMAYER, L. (2000). Valuing families: social work practice with families from a strengths perspectives. Social Work, 45(2), 118-130.

ESCAlAdA, M.; FERnÁNDEZ, S. y Fuentes, M. P. (2001). El diagnóstico social. Proceso de conocimiento e intervención profesional. Buenos Aires: Espacio Editorial.

Escartín, M., PAlOMAR, M. y SuÁREZ, E. (1997). Introducción al Trabajo Social II. Trabajo social con individuos y familia. Alicante: Aguaclara.

GARCíA, G. (2008). Reflexiones y utilidades sobre el diagnóstico y la programación en la intervención social. Ecos de Mary Richmond. Revista de Servicios Sociales y Politica Social, 10-38. 
GOBIERNO VASCO. (2006). Diagnóstico e Intervención Social. Herramienta informática para el Trabajo Social. Obtenido de http://www.euskadi.eus/gobierno-vasco/ contenidos/libro/diagnostico_1_soc/es_diagnost/adjuntos/Diagnostico.pdf

GOBIERNO VASCO. (2011). Instrumento para la valoración de las situaciones de riesgo y desamparo en los servicios sociales municipales y territoriales de la atención a la infancia y la adolescencia en la Comunidad Autónoma Vasca. Obtenido de http:// www.gizartelan.ejgv.euskadi.eus/r45\%20contss/es/contenidos/informacion/ balora/es_doc/adjuntos/Balora\%20C.PDF

GOBIERNO VASCO. (2012a). Instrumentos comunes de diagnóstico social y valoración de la exclusión. Obtenido de http://www.gizartelan.ejgv.euskadi.net/ r45-contss/es/contenidos/informacion/instrumento_valoracion/es_instrume/ instrumento_valoracion.html

Gobierno VAsco. (2012b). Modelo global de diagnóstico social. Obtenido de http:// www.gizartelan.ejgv.euskadi.eus/contenidos/informacion/instrumento_ valoracion/es_instrume/adjuntos/Modelo\%20diagnostico\%20social-junio2012.pdf

GOBIERNO VASCO. (2012c). Instrumentos comunes de diagnóstico social y valoración de la exclusión. Terminología y criterios. Ámbito económico. Obtenido de http:// www.gizartelan.ejgv.euskadi.eus/contenidos/informacion/instrumento_ valoracion/es_instrume/adjuntos/1-MANUAL\%20economico.pdf

GOBIERNO VASCO. (2012d). Instrumentos comunes de diagnóstico social y valoración de la exclusión. Terminología y criterios. Ámbito convivencial. Obtenido de http://www.gizartelan.ejgv.euskadi.eus/contenidos/informacion/instrumento_valoracion/es_instrume/adjuntos/2-MANUAL\%20convivencial.pdf

GOBIERNO VASCO. (2012e). Instrumentos comunes de diagnóstico social y valoración de la exclusión. Terminología y criterios. Ámbito personal. Obtenido de http:// www.gizartelan.ejgv.euskadi.eus/contenidos/informacion/instrumento_ valoracion/es_instrume/adjuntos/3-MANUAL\%20personal.pdf

GOBIERNO VASCO. (2012f). Instrumentos comunes de diagnóstico social y valoración de la exclusión. Terminología y criterios. Ámbito de la salud. Obtenido de http:// www.gizartelan.ejgv.euskadi.eus/contenidos/informacion/instrumento_ valoracion/es_instrume/adjuntos/4-MANUAL\%20salud.pdf

GOBIERNO VASCO. (2012g). Instrumentos comunes de diagnóstico social y valoración de la exclusión. Terminología y criterios. Ámbito social. Obtenido de http:// www.gizartelan.ejgv.euskadi.eus/contenidos/informacion/instrumento_ valoracion/es_instrume/adjuntos/5-MANUAL\%20social.pdf

GREENWOOD, E. (1955). Social science and social work. A theory of their relationship. Social Service Review, XXIX, 28.

Hamilton, G. (1923). Progress in Social Case Work. Some changes in Social Case Work. Obtenido de National Conference on Social Welfare. Official 
proceedings of anual meeting: http://quod.lib.umich.edu/n/ncosw/ACH8650. 1923.001/347? rgn=full+text;view=image

Hamilton, G. (1946). Principles of Social Case Recording. New York: The New York School of Social Work by Columbia University Press.

Hamilton, G. (1951). Teoría y práctica del trabajo social de casos. México: Editorial Médica Mexicana.

Hollis, F. (1954). Casework diagnosis. What and why? Smith College Studies in Social Work, 24(3), 1-8.

ItuARTE, A. (1992). Procedimiento y proceso en Trabajo Social Clínico. Madrid: Consejo General de Colegios Oficiales de Diplomados en Trabajo Social y Asistentes Sociales.

KARLS, J. y O'KeFFE, M. E. (2008). Person-In-Environment-System Manual (2. ${ }^{a}$ ed.). Washington D.C.: National Asociation of Social Workers.

Karls, J. y Wandrei, K., ED. (2008). Person-In-Environment System. The PIE Clasification System for Social Functioning Problems (1994, 1. ${ }^{a}$ ed.). New York: National Asociation for Social Workers.

KHISTARDT, W. (1994). An empowerment agenda for case management research: Evaluating the strengths model from the consumer's perspective. En M. Harris, H. Bergman, \& (Eds.), Case managements for mentally ill patients: Theory and practice (pp. 112-125). Langhorne, PA.: Harwood.

Ley 12/2008, de 5 de diciembre, de servicios sociales de la Comunidad Autónoma del País Vasco, Boletín Oficial del País Vasco, 246, de 24 de diciembre de 2008.

Martín, M., Miranda, B. y SuÁrez, E. (1996). Manual de Indicadores para el Diagnóstico Social. Vizcaya: Colegios Oficiales de Diplomados en Trabajo Social de la Comunidad Vasca.

Payne, M. (1995). Teorías contemporáneas del Trabajo Social. Barcelona: Paidós. PeLtON, G. I. (1910). The history and status of hospitall social work. Recuperado el 2011, de National Conference of Social Welfare Proceedings: http://quod.lib. umich.edu/n/ncosw/ACH8650.1910.001/351?rgn=full+text;view=image

Perlman, H. (1957). Social casework: A problem solving process. Chicago: University Press.

Perlman, H. (1961). The role concept and social casework: some explorations. Social Service Review, 370-381.

Perlman, H. (1968). Social casework: A problem solving process. Chicago: University Press.

Rank, O. (1936). Will therapy. New York: Alfred. A. Knopf.

RAPP, C. (1998). The strengths model: Case management with people suffering from severe amd persistent mental illness. New York: Oxford University Press. 
RichmOND, M. (1917). The social case worker's task. Obtenido de National Conference of Social Welfare Proceedings: http://quod.lib.umich.edu/n/ ncosw/ACH8650.1917.001/137? rgn=full+text;view=image

Richmond, M. (2007). Diagnóstico social (1. a ed., 1917, Titulo original: Social Diagnosis. Traducción; TRIDIOM ed.). Madrid: Siglo XXI.

RoBinson, V. (1930). A changing Psychology in Social Case Work. Chapel Hill: The University of North Carolina Press.

Rubin, A., \& BABBiE, E. (1997). Research Methods for Social Work. London: International Thomson Publishing Europe.

SAinsbury, E. (1970). Social Diagnosis in Casework. (N. Timms, Ed.) London: Routledge \& Kegan Paul.

SAlebeEY, D. (1996). The strengths perspective: Extensions and cautions. Social work, 41(3), 296-305.

SAlebeEY, D. (Ed.). (1992). The strenghts perspective in Social Work Practice (2. ${ }^{a}$ ed.). New York: Longman.

SCARÓN DE QUINTERO, M. T. (1974). El diagnóstico social. Buenos Aires: Humanitas.

SELBY, L. (1958). Typologies for caseworkers: Some considerations and problems. Social Service Review, XXXII, 341-349.

TIMMS, N. P. (1964). Social casework. London: Routledge \& Kegan Paul.

TURNER, F. (Ed.). (1968). Differential diagnosis and treatment in social work. New York: The Free Press.

TURNer, F. (2002). Diagnosis in Social Work: New imperatives. New York: The Haworth Press.

TURNER, F. (Ed.). (1974). Social work treatment: Interlocking theroretical aprproaches. New York: The Free Press.

Weick, M., RaPP, C., Sullivan, W. y KisthardT, W. (1989). A strengths perspective for social work practice. Social work, 34(6), 350-354.

Woods, E. y Hollis, F. (1964). Casework. A psychosocial therapy (5. ${ }^{a}$ ed.). New York, United States of America: McGraw-Hill Higher Education. A Division of The MacGraw-Hill Companies. 